\title{
Efectos de un programa de entrenamiento de Hypopressive RSF Reprogramación Sistémica Funcional en mujeres sanas Effects of a Hypopressive Exercise training program on healthy women \\ *Ignacio Matías Barresi Leloutre, **Óscar Hernández Vian \\ *Global Experience \& Sport Club (España), **Instituto Catalán de Salud (España)
}

\begin{abstract}
Resumen. La incorporación de la Gimnasia Abdominal Hipopresiva (GAH) en los centros de fitness está en auge, se trata de una técnica postural global y sistémica que consigue una disminución de la presión intratorácica, abdominal y pélvica, que ayuda a mejorar la tonificación del suelo pélvico y de la musculatura abdominal y a disminuir el perímetro abdominal. El objetivo del presente estudio consiste en describir los efectos de un programa de entrenamiento de ocho semanas de ejercicio hipopresivo en mujeres sanas de la población general. Se trata de un estudio pre experimental de un solo grupo al que se le realiza medidas pre y post intervención. El estudio se realizó en la comarca del Vallès Occidental de Barcelona durante el 2016 y 2017. Participaron mujeres sanas que acudieron a los diferentes grupos organizados. Intervención: ocho semanas de entrenamiento hipopresivo de 30 minutos de duración, dos días por semana. Tras la realización de la intervención se produjo una disminución media del perímetro abdominal de $3.48 \mathrm{~cm}(\mathrm{p}=.006)$ y de una mejora en el flujo espiratorio máximo de $40 \mathrm{l} / \mathrm{min}$ ( $\mathrm{p}=.021$ ). Por lo tanto, un entrenamiento continuado de 8 semanas de duración reduce el perímetro abdominal mejorando el tono muscular y mejora el flujo respiratorio máximo, y en consecuencia mejora la capacidad respiratoria de las participantes.
\end{abstract}

Palabras clave: ejercicio hipopresivo; gimnasia abdominal hipopresiva; perímetro abdominal; flujo espiratorio máximo; evaluación.

\begin{abstract}
More and more fitness centers are now incorporating to their service offer hypopressive exercises, focused on global and systemic postural improvement/education. Hypopressive exercise helps reducing intrathoracic, abdominal, as well as pelvic pressure, thus helping to improve the tone of the pelvic floor and abdominal muscles, and eventually reducing abdominal perimeter. The goal of this study is to describe the effects of an eight-week hypoppressive exercise program on a group of healthy women from the Western Vallés Region of Barcelona during 2016-17. A single-group pre-post test approach was used. The program consisted of two 30-minute hypopressive training sessions per week, over the eight-week duration of the program. Abdominal perimeter was reduced on an average of $3.48 \mathrm{~cm}$ while Peak Flow was increased up to an average of $40 \mathrm{l} / \mathrm{min}$. Sustained hypopressive exercise over 8 weeks (significantly) reduces abdominal perimeter and improves muscle tone and Peak Flow, therefore increasing trainees' breathing/respiratory capacity. Keywords: Hypopressive exercice; Hypopressive Abdominal Fitness; abdominal perimeter; Peak Flow; assessment.
\end{abstract}

\section{Introducción}

Cada vez son más los centros de fitness que incorporan la práctica de la gimnasia abdominal hipopresiva $(\mathrm{GAH})$ como una alternativa de actividad física saludable (Álvarez, Rial, Chulvi, García \& Cortell, 2016). Actualmente, estos centros presentan un entorno actual cada vez más tecnificado, que convive con actividades físicas dirigidas con aplicaciones que colaboran en la práctica de ejercicio físico. Las clases dirigidas requieren de las indicaciones de un instructor para poder desempeñarse correctamente, y estas no podrán ser substituidas por la tecnología (Estrada, Sánchez, Són-Grima, $\&$ Casteral, 2020). De hecho los resultados sugieren que el ejercicio físico dirigido es más efectivo que el prescrito mediante una aplicación móvil (Muntaner, Palao, \& Vidal, 2016). Una de estas actividades son los ejercicios hipopresivos. Esta gimnasia fue diseñada a finales de los 80 por Marcel Caufriez y tenía como objetivo conseguir una mejora de la tonificación abdominal en el postparto sin alterar la estática del suelo pélvico. (Caufriez, 1997). Posteriormente, se adaptó la GAH al mundo del fitness creándose la Hypopressive RSF (Reprogramación Sistémica Funcional), con el objetivo de acercar la actividad al público en general y a de la promoción de la salud en particular (Rial, Villanueva \& Fernández, 2011). Pero este auge cada vez más creciente contrasta con la falta de estudios que evalúen sus beneficios en la salud y el bienestar (Martín Rodríguez \& Bo, 2019).

Fecha recepción: 16-07-19. Fecha de aceptación: 25-05-20 Ignacio Matías Barresi Leloutre

ignabarresi@gmail.com
Según el propio autor del método, la GAH se define como una técnica postural y sistémica que supone una activación de diferentes paquetes musculoesqueléticos que son antagonistas del diafragma desde un punto de vista postural. Con esta técnica se consigue provocar una disminución de la presión intratorácica y abdominal (Caufriez, 1997; Flores, \& Uclés, 2018; Rial, et al., 2011).

La práctica de la GAH se basa en la realización de ejercicios respiratorios con un adecuado trabajo postural. Concretamente son cinco postulados básicos los que nos aporta los beneficios: la autelongación, el doble-mentón, la decoaptación de la articulación glenohumeral, anteposición del eje axial y la respiración costo-diafragmática.

-Autoelongación: consiste en un estiramiento axial de la columna vertebral que provoca una tensión de los músculos espinales profundo y de los músculos extensores de la espalda.

- Doble-mentón o autolongación cervical: consiste en la colocación de la columna cervical disminuyendo la lordosis de esta zona a través de una ligera retracción del mentón provocando un movimiento de la cabeza hacia el punto vértex o coronilla.

- Decoaptación de la articulación glenohumeral: se provoca realizando una abducción de las escápulas que implican un aumento de la circunferencia superior de la parrilla costal, causada por la activación de los músculos serratos.

-Anteposición del eje axial: se trata de un desequilibrio el eje longitudinal antero-posterior que implica una variación en el centro de gravedad en dirección ventral como causa de la activación de la musculatura paravertebral. 
- Respiración costo-diafragmática: durante la «fase de inspiración normal» se incrementa el volumen de la caja torácica y se reduce la presión en ella a causa de la apertura de la costilla hacia afuera y arriba, aumentando el diámetro transverso y antero-posterior de la misma, lo que permite la expansión pulmonar y la entrada del aire. (Caufriez, 1997).

Teniendo en cuenta esta premisa, la GAH consiste en la realización de diferentes ejercicios dinámicos y estáticos que favorecen la activación refleja de esta musculatura abdominal mediante la adquisición de diferentes posturas en isometría y/o contracción.

Según el autor del método hipopresivo, las contraindicaciones para la práctica de esta gimnasia son: la hipertensión arterial sin control médico, el embarazo, enfermedades obstructivas respiratorias severas, enfermedades cardiovasculares (Caufriez, 1997). La revisión de la literatura publicada muestra diferentes beneficios que se podría mejorar con la realización de un programa de ejercicios hipopresivos, entre ellos se destacan los siguientes:

Uno de los aspectos a considerar a la luz de los artículos publicados es la mejora de la tonificación del suelo pélvico. Según un estudio realizado por Álvarez et al. (2016) se encontraron mejoras en la contractilidad y tonificación de la musculatura del suelo pélvico respecto al grupo al que no había hecho la práctica de la gimnasia hipopresiva, presentando diferencias estadísticamente significativas $(p<.05)$. Anteriormente, Teixeira, Magalhäes, Stupp, Oliveira, Aquino \& Jarmy (2012), reportaron una mejora de la tonificación de la musculatura del suelo pélvico después de realizar un programa de gimnasia hipopresiva para el tratamiento del prolapso pélvico en mujeres, con diferencias estadísticamente significativas $(p<.001)$ respecto al grupo que no había realizado ningún tratamiento. Así mismo, en los estudios de Bernardes, et al. (2012) y de Bø, Mørkved, Frawley, \& Sherburn (2009) se remarca que el ejercicio hipopresivo mejoró la tonificación del suelo pélvico y de la musculatura transversa abdominal. Además es necesario añadir que en un estudio realizado per Tracogna, \& Rial (2018), la expectativa más esperada con un $85 \%$ de las mujeres participantes en el estudio, fue la mejora de la tonificación del suelo pélvico y de la recuperación de la incontinencia urinaria.

Otro de los aspectos a considerar es la mejora de la fuerza de la musculatura abdominal. El hecho de que el ejercicio hipopresivo sea una técnica postural sistémica estimula la reprogramación de la contractilidad de las fibras abdominales con la consecuencia de una mejora en la fuerza de la contractilidad de éstas (Caufriez, 2014). Así mismo, el entrenamiento de la musculatura del suelo pélvico mejora la fuerza de la musculatura abdominal (Flores, \& Uclés, 2018). Así mismo, en un estudio de Da Cuña, Soto, González, \& Latarón (2018), señalaron que mujeres deportistas tras la práctica de ejercicio hipopresivo, mejoraba la muscular abdominal al medirse a través de ecógrafo.

Otro factor a considerar es la mejorar de la actividad contráctil del diafragma. Este hecho favorece por la respiración costo-diafragmática utilizada en el método y por el posicionamiento necesario, ya que, se disminuye la presión intratorácica y abdominal. Caufriez ha hecho diferentes medidas con electromiografía para constatar esta activación (Caufriez, 2014).
Otro factor que se ha conseguido una mejora según diferentes publicaciones es sobre la incontinencia urinaria. En un estudio realizado por Esparza en 2007, en el que se aplicó un protocolo de gimnasia abdominal hipopresiva a un grupo de 100 mujeres con incontinencia urinaria e hipotonía del suelo pélvico, demostró un aumento en la fuerza contráctil $(20 \%)$ en el tono muscular base (58\%) y en el tono de carga (48\%) (Esparza, 2007). En otra publicación sobre la prevención de la incontinencia urinaria postparto, se encontró que incrementaba el tono de base y la carga de la musculatura del suelo pélvico, mejoraba el bloqueo perineal al esfuerzo, y disminuía o desaparecía la incontinencia urinaria (Sánchez, González \& Hernández, 2014). De la misma manera, Palacios, \& Díaz (2016), señalan que la fisioterapia obstrética tanto preventiva como de tratamiento mejora la incontinencia urinaria postparto, dentro de estas técnicas se recoge el entrenamiento con ejercicios hipopresivos. Por otra parte, en un estudio realizado por Rial (2014), un programa de doce semanas de gimnasia hipopresiva disminuye la severidad de síntomas de la incontinencia urinaria. Así mismo, Baruc, Rial, Chukvi, \& Fabre (2017), obtienen que tras la práctica de un ejercicio hipopresivo supervisado las mujeres multíparas participantes tuvieron un disminución en el índice de las pérdidas de orina y en el grado de severidad de la incontinencia.

Otro de los elementos a considerar sería la reducción del perímetro abdominal. Diferentes publicaciones reportan una mejora en este aspecto. Se destaca el estudio publicado el 2014 en el que después de realizar un programa de gimnasia hipopresiva a 10 mujeres y compararlas con 10 mujeres del grupo control se logró una disminución de $1.79 \mathrm{~cm}$ de cintura abdominal, estas diferencias fueron estadísticamente significativas. Es importante aclarar que produce una reducción del perímetro abdominal pero no del índice de masa corporal que se mantiene estable (Soriano, González-Millán \& Salinero, 2014). Hay que tener en cuenta que el perímetro abdominal es una de las variables que más se vincula con el riesgo cardiovascular, de ahí su importancia (Rodríguez, Cabrera, Aguirre-Jaime, Domínguez, Brito \& Almeida, 2010).

Otro de los aspectos es la mejora de la estática de la columna vertebral. En el estudio realizado por Galindo \& Espinoza (2009), sobre un análisis de diferentes programas de ejercicio en la lumbalgia mecanopostural, demostró que el grupo que hizo un entrenamiento con hipopresivos y el grupo de Pilates son los que más mejoraron la flexibilidad de la columna lumbar, y que el tiempo de amplitud y de activación también mejora en ambos grupos. Por otro lado un estudio realizado en mujeres jugadoras de fútbol después de realizar un programa de gimnasia hipopresiva de 12 sesiones mostró una repercusión sobre la extensibilidad isquiosural y la movilidad lumbar. Se encontraron mejoras en ambos aspectos con unas diferencias estadísticamente significativas $(p<.05)$ (Rial, Negreira, Álvarez \& García, 2013). Anteriormente, en una publicación de 2006 demostró después de practicar durante 10 sesiones de una hora de duración, variaciones significativas respecto al grupo control en la estática vertebral (flecha cervical: $p=.002$; dorsal $p=.0001$; lumbar $p=.006$ ). En la posición del eje occipital $(p=.04)$; y en la medida de flexibilidad de la columna, principalmente en la flexión de tronco $(p=.005)$. Así mismo, se produjo una mejora en la 
fuerza isométrica de los músculos extensores del tronco: test de Sorensen-Biering $(p=.02)$ (Caufriez, Fernández, Fanzel \& Snock, 2006).

Por el contrario no se han encontrado publicaciones que relacionen la práctica de la GAH y la tonificación de la musculatura respiratoria, a pesar de ser uno de los aspectos básicos en el desarrollo de esta técnica. Lo que sí se hay publicado es un artículo que relaciona el entrenamiento de la fuerza abdominal con la mejora de la función respiratoria (Rodríguez, Alarcón, Gutiérrez, Hermosilla, Contreras, Baéz, 2014). Teniendo en cuenta este hecho consideramos que debe será una variable a tener en cuenta cuando se pretenda valorar el impacto del ejercicio hipopresivo.

Son escasos relativamente escasos los estudios publicados que evalúen el impacto de la gimnasia abdominal hipopresiva tanto a nivel internacional como nacional (Álvarez, et al., 2016; Rial, et al., 2015, Soriano et al., 2014; Texeiran et al., 2014; Caufriez, et al., 2006). Son mucho menos los estudios encontrados que evalúen el impacto sobre la disminución de la cintura abdominal (Rodríguez et al., 2010), y no se han encontrado estudios que valoren el efecto sobre la mejora de musculatura respiratoria medida a través del flujo espiratorio máximo (FEM). Por este motivo será importante la realización de este tipo de estudio para abrir una nueva línea de investigación de contemple este tipo de variables.

Como objetivo general se planteó el hecho de analizar los efectos de un programa de entrenamiento de ocho semanas de ejercicio hipopresivo en mujeres sanas de la población general. Como objetivos específicos se especificaron:

Analizar las diferencias en el perímetro abdominal en los participantes en un programa de entrenamiento de Gimnasia Abdominal Hipopresiva de ocho semanas de duración en mujeres sanas de la población general.

Analizar las diferencias en el flujo espiratorio máximo en los participantes en un programa de entrenamiento de Gimnasia Abdominal Hipopresiva de ocho semanas de duración en mujeres sanas de la población general.

\section{Material y Métodos}

Se diseñó un estudio pre experimental prospectivo, con medidas pre y post test para un solo grupo de intervención. El estudio se realizó en la comarca del Vallès de la provincia de Barcelona en 2016 y 2017. Los sujetos de estudio fueron mujeres sanas que participaron en dististos grupos de entrenamiento en ejercicios hipopresivos (nunca más de ocho ) organizados en diferentes localidades de la provincia de Barcelona.

\section{Criterios de inclusión}

Mujeres mayores de 18 años que participaron en un mínimo de ocho sesiones de entrenamiento de ejercicio hipopresivo, que se considera como tiempo mínimo para notar beneficios.

Disponer de consentimiento informado.

\section{Criterios de exclusión}

Mujeres que presenten algún tipo de contraindicación para la realización de ejercicio hipopresivo: HTA sin control médico, el embarazo, enfermedades obstructivas respiratorias severas, enfermedades cardiovasculares.

Haber participado en menos de ocho sesiones de entrenamiento de ejercicio hipopresivo.

No poder realizar correctamente el peak-flow.

Realizar dieta hipocalórica.

\section{Intervención de ejercicios hipopresivos Basasdo en pos- tulados de Marcel Caufriez}

El protocolo de intervención de ejercicio hipopresivo que fue aplicado, constó de ocho semanas de entranamiento en la que se realizaron dos sesiones de 30 minutos a la semana. Todos los ejercicios propuestos estaban basados en los siguientes aspectos: (1) Patrón respiratorio hipopresivo: de tres ciclos, de dos tiempos de inspiración y cuatro tiempos de espiración máxima, hasta conseguir en la última espiración, la sensación de vaciado completo. (2) Posicionamiento: anteposición del eje, decoaptación de la cintura escapular con la consecuente activación fásica del serrato anterior y autoelongación de la columna vertebral.

La primera sesión se dedicó exclusivamente a trabajar los patrones posturales anteriormente propuestos de : autoelongación, doble mentón o elongación cervical, decoaptación de la articulación glenohumeral y adelantamiento del eje axial, respetando el patrón respiratorio anteriormente mencionado.

En la segunda sesión se introdujeron ejercicios estáticos con la intención de mecanizar el control postural global, utilizándose las siguientes variantes: Ejercicios en pared, conocido como preparación al Atlas. Posteriormente se realizó un ejercicio en bipedestación conocido como posición Aurora, y un ejercicios en cuadrupédia, conocido como The Horse.

En la tercera sesión, se realizaron ejercicios estáticos, con el fin de mantener y controlar la postura; todos con la pauta de realizar tres ciclos respiratorios completos, hasta llegar a la situación de espiración máxima, y una vez comenzada la apnea, el foco de atención se dirige a mantener el posicionamiento, con autoelongación máxima, activación fásica del Serrato mayor a través de la decoaptación, y mantener la anteposición del eje en todos los ejercicios, excepto en la posición decúbito supina.

A partir de la cuarta sesión se introdujeron ejercicios dinámicos y se procedió a la combinación con los ejercicios estáticos hasta completar la intervención de ocho semanas (tabla 1).

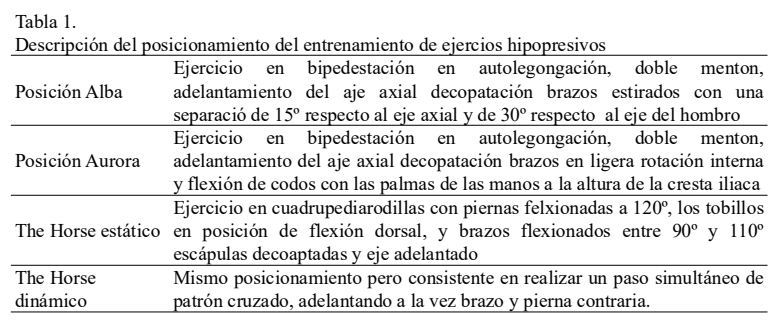

Todas las personas que han participado en el estudio leyeron y firmaron el consentimiento de participación en la investigación, cuyo procedimiento se realizó respetando los principios éticos de la declaración de Helsinki y la ley de protección de datos 12/2018. Se les explicó las características del estudio y tuvieron la oportunidad de abandonarlo en caso de creerlo oportuno. Durante la primera sesión firmaron 
dicho consentimiento informado y se familiarizaron con los ejercicios del programa de estudio.

\section{Variables de estudio}

Variable independiente: intervención de ocho semanas de ejercicios hipopresivos.

- Variables dependientes:

- Perímetro abdominal: tomada en la zona delimitada por el borde subcostal y la cresta ilíaca, pasando la cinta métrica por la zona umbilical del abdomen, en posición ortoestática y en reposo. Las mediciones se expresaron en centímetros.

- Flujo Espiratori Máximo (FEM): medido a través del peak-flow Sibelmed modelo datospir PEAK-10, medido en litros/minuto.

- Variables de control: edad, número de sesiones realizadas.

\section{Análisis estadístico}

Para el análisis univariante se han utilizado los estadísticos descriptivos de medida de tendencia central y de dispersión. Se ha comprobado la normalidad de las variables numéricas con el test de kolmogorov-Smirnov. Para el análisis bivariante pre-test i post-test se ha utilizado la t de Student para muestras relacionadas o test de Wilcoxon, para el análisis de asociación se ha utilizado en test de correlación de Spearman. Se ha asumido en todos los casos un nivel de confianza del 95\% $(p<.05)$.

\section{Resultados}

Han completado un total de ocho sesiones de hypopressive RSF. de 16 mujeres en 2016 y 2017. Cinco mujeres fueron descartadas, dos por no realizar las ocho sesiones y tres por presentar sintomatología respiratoria que invalidaba el uso del peak-flow.

La media de edad fue de 40.88 años +8.55 , con un mínimo de 23 años y un máximo de 63 años (tabla 2).

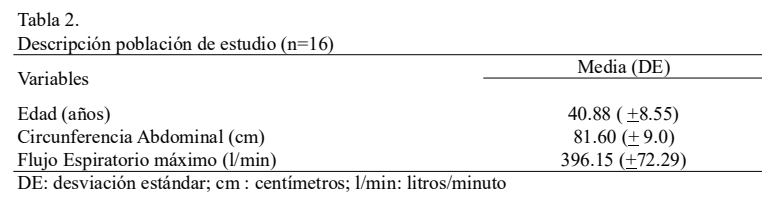

Respecto a la medida del perímetro abdominal antes de participar en las sesiones de ejercicio hipopresivo fue de $81.60 \mathrm{~cm}+9.0$, mientras que después de realizarlas fue de $78.12 \mathrm{~cm}+9.2$. Por tanto se redujo de media un total de 3.48 $\mathrm{cm}$.

El análisis bivariante mediante el test de Wilconxon muestra que tras la realización de la sesiones se han obtenido una reducción de la cintura abdominal, siendo estas diferencias estadísticamente significativas $(p=.006)$ (tabla 3$)$.

Tabla 3.

Mediciones antes y después del programa de ejercicio para las variables de estudio

\begin{tabular}{lcccc} 
Variables & Media (DE) Pres & Media (DE) Post & $\mathrm{Z}$ & $p^{*}$ \\
\hline Circunferencia Abdominal $(\mathrm{cm})$ & $81.60(+9.0)$ & $78.12(+9.2)$ & -2.76 & 0.006
\end{tabular} Fluio Espiratorio Méximo (FEM) (1/min) $\quad 396.15(+7229) \quad 436.15(+77.19) \quad-2.31 \quad 0.021$ \begin{tabular}{lllll} 
Flujo Espiratorio Máximo (FEM) (1/min) & $396.15( \pm 72.29)$ & $436.15( \pm 77.19)$ & -2.31 & 0.021 \\
\hline DE: desviación estánda; Z
\end{tabular} DE: desviación estándar; Z: test Wilcoxon; p:significación estadística ${ }^{*} p=.05$; cm: centímetros;

Respecto a la medición del flujo espiratorio máximo antes y después de realizar las sesiones de ejercicios hipopresivos, es necesario remarcar que tres mujeres fueron descartadas por presentar sintomatología respiratoria (resfriado común) que dificultaba la realización de la técnica correctamente y por tanto invalidaba el resultado obtenido. La media del FEM previo a la ejecución de las diferentes sesiones de ejercicios hipopresivos fue de $396.15 \mathrm{l} / \mathrm{min}+72.29$ y tras la realización de las sesiones fue de $436.15 \mathrm{l} / \mathrm{min}+77.19$, mejorando una media de $40 \mathrm{l} / \mathrm{min}$ estas diferencias fueron estadísticamente significativas $(p=.021)$ (tabla 3$)$.

Respecto al análisis de correlación de Spearman para relacionar la disminución del perímetro abdominal y la mejora del FEM las diferencias no fueron estadísticamente significativas. ( $p>.05)$, así como la correlación entre la edad y perímetro abdominal y la edad y FEM.

\section{Discusión}

Los efectos de un programa de entrenamiento de ocho semanas de ejercicio hipopresivo en las mujeres que han participado en el presente estudio se centra en la reducción de la cintura abdominal y en la mejora de la musculatura respiratoria.

En cuanto a la media de edad del presente estudio fue mayor que la de otras publicaciones (Álvarez, et al., 2016; Rial, et al., 2015, Soriano, et al., 2014; Rodríguez, et al., 2010). Ya que en ellos se eligió población que practicaba deporte profesionalmente, mientras que la presente muestra era de mujeres de población general.

Respecto a la disminución de la cintura abdominal se debe tener en cuenta que la gimnasia abdominal mejora la tonificación de la musculatura profunda (Stupp et al, 2011) este hecho y en particular en el presente estudio se ha reducido el perímetro abdominal en una media de $3.48 \mathrm{~cm}$. Esta reducción ha sido ligeramente superior a la realizada en otros estudios como el de Álvarez et al. (2016), con $2.89 \mathrm{~cm}$ o el de Soriano et al. (2014), que presenta una reducción de $1.79 \mathrm{~cm}$. Se debe tener en cuenta que ambos estudios presentan una población más joven concretamente 23.36 años para el primer estudio y 21.11 años para el segundo. Además la media de cintura abdominal también fue superior en el presente estudio, y por lo tanto, presentaba una mayor posibilidad de mejora. Así mismo Rial et al. (2014), también obtuvo una diferencia estadísticamente significativa en la reducción del perímetro abdominal. En esta ocasión también se trataba de una muestra de estudio compuesta por mueres profesionales del deporte.

Respecto a la mejora en la musculatura respiratoria medida a partir del flujo espiratorio máximo no se dispone de publicaciones previas para comparar los resultados obtenidos. Se debe tener en cuenta que con la realización de la gimnasia hipopresiva se ejercitan los músculos respiratorios, y este hecho ha provocado una mejora en la fuerza de los músculos respiratorios, dato constatado por la mejora en el valor medio del FEM en 401/min. Además y aunque no se realizó un medida expresa en las participantes, éstas constataron un mejora del tiempo de apnea. En las primeras sesiones la mayoría de las participantes no sobrepasaban los 5 segundos, mientras que al final era superior a los 10 segundos. Todos estos aspectos llevan a confirmar la mejora en la fuerza de musculatura respiratoria. La única publicación que se encontró que valora la mejora del FEM tras la realización 
de un entrenamiento de músculos abdominales. Específicamente no se trataba de un entrenamiento hipopresivo, sino de fuerza abdominal. La mejora media en el FEM fue muy similar a la del presente estudio, concretamente de $35.3 \mathrm{l} / \mathrm{min}$. Debemos tener en cuenta que la población del estudio mencionado se trataba de población adolescente tanto de hombre como mujeres a diferencia del presente estudio que se trata de mujeres de población general.

\section{Conclusiones}

Teniendo en cuenta los resultados obtenidos en el presente estudio podemos concluir que un programa de ocho semanas de ejercicio hipopresivo por mujeres sanas reduce el perímetro de la cintura abdominal y el flujo respiratorio máximo, por lo tanto la fuerza de la musculatura respiratoria accesoria, mejorando por tanto el tono muscular. Otro de los efectos que produjo fue la mejora del flujo respiratorio máximo, y por lo tanto de capacidad respiratoria de los participantes.

Por lo tanto, se recomienda la práctica de ejercicio hipopresivo como una opción adecuada para la mejora de la respiración y para reducción de la cintura abdominal. Serán necesarios más estudios experimentales que corroboraren otros aspectos que puedan mejorar la GAH en otros grupos de población, con una muestra más amplia, que incorporen a hombres y que recojan más datos sobre el impacto de la GAH sobre la musculatura respiratoria.

\section{Limitaciones}

Como limitaciones de estudio destaca el tamaño de la muestra, todo y que la mayoría de los estudios publicados presentan muestras inferiores a 20 individuos, sería recomendable aumentar este número. En nuestro caso y a diferencia de otras publicaciones se ha elegido un rango de edad mucho mayor por lo que nos da la idea de qué puede pasar con un rango de edad mayor pudiéndose extrapolar a una población general, no en jóvenes como la mayoría de los estudios previos consultados. Además sería adecuado incorporar hombres para valorar el efecto de este tipo de entrenamiento.

\section{Referencias}

Alvárez, M. M., Rial, T., Chulvi „I., García, J. L. \& Cortell „J.M. (2016) ¿Puede un programa de 8 semanas basado en la técnica hipopresiva producir cambios en la función del suelo pélvico y composición corporal de jugadoras de rugby? Retos. Nuevas Tendencias en Educación Física, Deporte y Recreación, 30, 26-29

Bernardes, B., Resende, A., Stüpp, L., Oliveira, E., Castro, R., Bella, Z., Girao, M., \& Sartori, M. G. (2012). Efficacy of pelvic floor muscle training and hypopressive exercises for treating pelvic organ prolapse in women: Randomized controlled trial. São Paulo Med J. 130 (1), 5-9.

Baruc, A., Rial, T., Chukvi, I. Fabre, P. (2017). Ejercicio hipopresivo en el tratamiento de la incontinencia urinaria en mujeres multíparas: serie de casos. RECIEN, Revista Científica de Enfermería,13, 36-53

Bø, K., Mørkved, S., Frawley, H., \& Sherburn, M. (2009). Evidence for benefit of transversus abdominis training alone or in combination with pelvic floor muscle training to treat female urinary incontinence: A systematic review. Neurourology and Urodynamics, 28(5), 368373

Caufriez, M. Gymnastique abdominale hypopressive, Bruselas:M.C. Editions; 1997.
Caufriez, M., Fernández, J. C., Fanzel, R. \& Snock, T. (2006). Efectos de un programa de entrenamiento estructurado de Gimnasia Abdominal Hipopresiva sobre la estática vertebral cervical y dorsolumbar. Fisioterapia, 28(4), 205-216

Caufriez, M. (2014). Caufriez concept. Apunts formatius nivell expert. Pàg. 36

Da Cuña, I., Soto, M., González, Y., Latarón, E.M. (2018). Activación de la musculatura abdominal a través de los ejercicios abdominales hipopresivos. Revista internacional de deportes colectivos. 34, 4255

Galindo, G. A. \& Espinoza, A. S. (2009). Programa de ejercicio en lumbalgia mecanopostural. Revista Mexicana de Medicina Física y Rehabilitación, 21, 11-19

Esparza, S. (2007). Gimnasia Abdominal Hipopresiva. Congreso franco español del suelo pelvico y pelviperineología. San Sebastian.

Estrada-Marcén, N., Sánchez-Bermúdez, J., Simón-Grima, J., \& CasteralSerad, J. (2020). Uso de dispositivos fitness por parte de usuarios de gimnasios. Retos. Nuevas Tendencias en Educación Física, Deporte y Recreación, 38, 26-32

Flores, M.G. \& Uclés, V., 2018. Ejercicios hipopresivos: prescripción, técnicas y efectividad. Revista clínica de la Escuela de Medicina. 1, 113

Martín-Rodríguez, S. \& Bo, K. (2019). Is abdominal hypopressive technique effective in the prevention and treatment of Pelvic floor dysfunction? Marketing or evidence from high-quality trials? $\mathrm{Br} J$ sports Med, 53(2), 135-136.

Muntaner, A., Palao, P. \& Vidal, J. (2016). Efectos de un programa de entrenamiento presencial vs prescripción a través de una aplicación móvil en personas mayores. Retos. Nuevas Tendencias en Educación Física, Deporte y Recreación, 29, 32-37

Palacios, A. \& Díaz, B. (2016). Fisioterapia en incontinencia urinaria de embarazo y posparto: una revisión sistemática. Clínica e Investigación en Ginecología y Obstetricia, 43(3), 122-131

Rial, T., Negreira, N., Álvarez-Sáez, M. \& García-Soidán J. L. (2015). ¿Puede un programa de ejercicio hipopresivo influir sobre la extensibilidad isquiosural y movilidad lumbar de jugadoras de futbol?. Revista de Preparación Física en el Futbol, 30, 41-50

Rial, T., (2014). Efectos de la gimnasia hipopresiva en la incontinencia urinaria de la mujer adulta. Revista española de educación física y deportes, 405(2), 93-94

Rial, T., Sousa, L., García, E., Pinsach, P. (2014) Efectos inmediatos de una sesión de ejercicios hipopresivos en diferentes parámetros corporales. Cuestiones de fisioterapia: revista universitaria de información e investigación en Fisioterapia, 43(1): 13-21

Rial, T., Villanueva, C. \& Fernández, I. (2011). Aproximación conceptual y metodológica al método hipopresivo. EF Deportes.com, revista digital, 162, 3-10

Rodríguez, I., Alarcón, M., Gutiérrez, C., Hermosilla, P., Contreras, T. \& Baéz, C., (2014). Efecto del entrenamiento de músculos abdominales sobre la función respiratoria en adolescentes sanos. Estudio Piloto. Revista Chilena de Enfermedades respiratoria, 30, 203-211

Rodríguez, M. C., Cabrera, A., Aguirre-Jaime, A., Domínguez, S., Brito, B. \& Almeida, D. (2010). El cociente perímetro abdominal/estatura como índice antropométrico del riesgo cardiovascular y diabetes. Revista medicina clínica (Barcelona), 134(9), 386-391

Sánchez, M. M., González, R. \& Hernández, E. (2014). Importancia de las técnicas hipopresivas en la prevención de la incontinencia urinaria postparto. Revista Científica de Enfermería, 8, 13-26

Soriano, L., González-Millan, C. \& Salinero, J. J. (2014). Efecto de un programa de gimnasia abdominal hipopresiva $(\mathrm{GAH})$ sobre el principal predictor de riesgo cardiovascular en una población de jóvenes sanos, sedentarios y nulípara. International Journal of Sport Sciences, 4(1), 36-45

Stüpp, L., Resende, A., Petricelli, C., Nakamura, M., Alexandre,S. \& Zanetti, M. (2011). Pelvic floor muscle and transversus abdominis activation in abdominal hypopressive technique through surface electromyography. Neurourology And Urodynamics ,30(8), 518521.

Teixeira, B., Magalhães, A. P., Stüpp, L., Oliveira, E., Aquino, R. \& Jármy, Z. I. (2012). Efficacy of pelvic floor muscle training and hypopressive exercises for treating pelvic organ prolapse in women: randomized controlled trial. Sao Paulo Medical Journal, 130(1), 5-9

Tracogna, V. \& Rial, T. (2018) Expectativas y beneficios percibidos del ejercicio hipopresivo por mujeres: una experiencia práctica. Retos. Nuevas Tendencias en Educación Física, Deporte y Recreación, 34, 138-141 